\title{
ERRATUM
}

\section{HER2/Neu: mechanisms of dimerization/oligomerization}

Patrick J Brennan, Toru Kumagai, Alan Berezov, Ramachandran Murali and Mark I Greene DOI: $10.1038 / \mathrm{sj} /$ onc1205119

Correction to: Oncogene (2000) 19, 6093-6101

In the above paper, the second author's surname was spelt incorrectly. It is correct as given above. 